\title{
Wissenschaftliche Taschenbücher
}

Eine Auswahl

lieferbarer Bände:

JOACHIM AUTH / DIETMAR GENZOW

KLAUS H. HERRMANN

Photoelektrische Erscheinungen

HANS BANDEMER

ANDREAS BELLMANN

WOLFHART JUNG / KIAUS RICHTER

Optimale Versuchsplanung

FRANK Berchelt

Prophylaktische Erneuerung

von Systemen

Joachim Bellach / Peter Franken

ELKE WARMUTH / WALTER WARMUTH Maß, Integral

and bedingter Erwartungswert

WOLFrAM BRaUer

HANS-WALDEMAR STREITWOLF

Theoretische Grundlagen

der Halbleiterphysik

SIEGFRIED BREHMER

Einfuihrung in die Maßtheorie

SIEGFRIED BREHMER

Hilbert-Räume und Spektralmaße

JOHN CUNNINGHAM

Vektoren

GEORG DAUTCOURT

Relativistische Astrophysik

K. Ch. Delokarov

Relativitätstheorie und Materialismus

WERNER DÜCK

Diskrete Optimierung
HANNELORE FISCHER

JOACHIM PIEHLER

Modellsysteme

der Operationsforschung

GOTTFRIED FrITZSOHE

Grundlagen und Entwurf

passiver Analogzweipole

Netzwerke I

Entwurf passiver Analogvierpole

Netzwerke II

HERBERT GOERING

Asymptotische Methoden zur Lösung von Differentialgleichungen

HERBERT GOERING

Elementare Methoden zur Lösung von Differentialgleichungsproblemen

Eduard Herlt / Nikolaus Salié Spezielle Relativitätstheorie

Helmut Hess

Der elektrische Durchschlag in Gasen

V. I. KaPPMAN

Nichtlineare Wellen

in dispersiven Medien

ULrich KaUsmanN

KraUs LommatzSCH

Františer NožičKa

Lineare parametrische Optimierung

A. R. KESSEL

Akustische Kernresonanz

KONRAD KREHER

Festkörperphysik

DieThr KRESS

Theoretische Grundlagen der Signalund Informationsübertragung 


\author{
V. S. LETOCHOW \\ Laserspektroskopie \\ DIERCK-EKKEHARD LIEBSCHER \\ Relativitätstheorie \\ mit Zirkel und Lineal \\ Wolfgang Meining \\ Digitalrechner \\ in der elektronischen Meßtechnik \\ Teil 1: Meßmethodik \\ Teil 2: Gerätetechnik \\ und Anwendungen
}

L. I. Miroschnitschenko

Kosmische Strahlen

im interplanetaren Raum

Peter Paufler

Gustav E. R. Schulze

Physikalische Grundlagen

mechanischer Festkörpereigenschaften

Teil I und II

ULRICH RÖSEBERG

Quantenmechanik und Philosophie

ALBRECHT ROST

Messung

dielektrischer Stoffeigenschaften

J. V. SAČKov

Wahrscheinlichkeit und Struktur

E. M. SAWIZKI

Perspektiven der Metallforschung

WOLFGANG SchäFER

Theoretische Grundlagen

der Stabilität technischer Systeme

ERNST SCHMUTZER

Symmetrien und Erhaltungssätze der Physik

VOLKMAR SCHURICHT

Kernexplosionen für friedliche $\mathrm{Z}$ wecke

NORBERT SIEBer

HANS-PETER LEIDHOLD

Einfuhrung in die Datenverarbeltung
GERHARD WUNSCH

Zellulare Systeme

Festkörperphysik

Entwicklungstendenzen und

Anwendungsmöglichkeiten

HORST MELCHER

Albert Einstein wider Vorurteile

und Denkgewohnheiten

Die Schöpfer der physikalischen Optik

Eine Artikelsammlung

HANS-GeORg SchöPF

Von Kirchhoff bis Planck

RENATE WAHSNER

Mensch und Kosmos

Die copernicanische Wende

Helmut Frifmer / Josef Brock

Grundlagen der Immunologle

EbERHARD HoFmanN

Funktionelle Biochemie des Menschen

Band 1 und 2

LOTHAR JÄGER

Grundlagen

der Klinischen Immunologie

KARLHEINZ LOHS

DieTer MaRTINeTZ

Entgiftung - Mittel, Methoden

und Probleme

JOAOHIM NitSOHMANN

Entwicklung bel Mensch und Tier

Stephan SchNitzLeR

Pharmakologische Aspekte

von Immunreaktionen

DIETER SPAAR

HELMUT KLEINHEMPFI

HANS JOACHIM MULLER

KuAUS NaUmaNe

Bakteriosen der Kulturpflanzen 
Eberhard Tedscher

Pharmakognosie

Teil I-III

Michael Theile

SIEGFRIED SCHERNECK

Zellgenetik

HEINRICH BREMER

Kuaus-Peter Wendlandt

Heterogene Katalyse

PeTer BirNer

HANS-JöRg HofmanN

Cornelius Weiss

MO-theoretische Methoden

in der organischen Chemie

GÜNTER EPPERT

Einführung

in die Schnelle Flüssigchromatographie

Gerhard Geiseler / Heinz Seidel

Die Wasserstoffbrückenbindung

HeLmut Hrapia

Einführung in die Chromatographie

HANS LUPPA

Grundlagen der Histochemie

Teil I und II

BURKart PhILIPP

GERHARD REINISCH

Grundlagen

der makromolekularen Chemie

Horst Remane / RaINeR HerzschuH Massenspektrometrie in der organischen Chemie

ADOLF ZsChUNKE

Kernmagnetische

Resonanzspektroskopie

in der organischen Chemie
Vorschau

auf die nächsten Bände:

HEINRICH ARNOLD

Physikalische Chemie der Halbleiter

HORST-HEINO voN BoRZESZKOWSKI

RENATE WAHSNER

Newton und Voltaire

WERNER DÖPKE

Dynamische Aspekte der Stereochemie organischer Verbindungen

GOTTFRIED FRITZSCHE

Entwurf aktiver Analogsysteme

Netzwerke III

Falko H. HerRmanN

MARINA HERRMANN

Das Hämoglobin des Menschen

GERHARD JACKISCH

Johann Heinrich Lamberts

„Cosmologische Briefe“ mit Beiträgen zur Frühgeschichte der Kosmologie

Das Neutron

Eine Artikelsammlung

VOLKER NOLLAU

Semi-Markovsche Prozesse

ROBERT ROMPE

HANS-JÜRGEN TREDER

Über Physik -

Studien zu ihrer Stellung

in Wissenschaft und Gesellschaft

RAINER SINZ

Chronopsychophysiologie,

Chịonobiologie und Chronomedizin

HUBERTUS STOLZ

Supraleitung 


\section{W'B}

BAND 191

Dieter Leuschner

Grundbegriffe der Thermodynamik

Mit 17 Abbildungen und 6 Tabellen

AKADEMIE-VERLAG - BERLIN 


\section{Reihe MATHEMATIK UND PHYSIK}

\section{Herausgeber:}

Prof. Dr. phil. habil. W. Holzmüller, Leipzig

Prof. Dr. phil. habil. A. Lösche, Leipzig

Prof Dr. phil. habil. H. Reichardt, Berlin

Prof. Dr. rer. nat. habil. H.-J. Treder, Potsdam

Verfasser:

Dr. rer. nat. Dieter Leuschner

Akademie der Landwirtschaftswissenschaften der DDR

ISBN 978-3-528-06855-4 ISBN 978-3-322-85665-4 (eBook)

DOI 10.1007/978-3-322-85665-4

\section{9}

Erschienen im Akademie-Verlag, 108 Berlin, Leipziger Str. 3-4

(c) Akademie-Verlag Berlin 1979

Softcover reprint of the hardcover 1st edition 1979

Lizenznummer: $202 \cdot 100 / 435 / 79$

Herstellung: VEB Druckhaus „Maxim Gorki“, 74 Altenburg

Bestellnummer: 7623408 (7191) · LSV 1144

DDR 8,- M 


\section{Geleitwort}

Die klassische Thermodynamik, geschaffen durch die Arbeiten vieler bedeutender Gelehrter, unter denen die deutschen Wissenschaftler eine besondere Stellung einnahmen, erfuhr durch ihre Anwendung in vielen Gebieten der Wissenschaft und Technik eine weite Verbreitung. Ungeachtet dessen besitzt die klassische Thermodynamik nur einen begrenzten Anwendungsbereich, da sie sich auf die Beschreibung der Gleichgewichtsprozesse beschränkt, der größte Teil der realen physikalischen, chemischen und biologischen Prozesse jedoch irreversibel verläuft. Gegenwärtig wird auf dem Gebiet der Thermodynamik der irreversiblen Prozesse mit wachsender Intensität gearbeitet. Es besteht die berechtigte Hoffnung, da $B$ in nicht allzu ferner Zukunft eine thermodynamische Theorie entstehen wird, welche die Mehrheit der in der Natur ablaufenden irreversiblen Prozesse umfaßt. Es ist deshalb erforderlich, die jungen heranwachsenden Wissenschaftler mit dem Problemkreis der Thermodynamik vertraut zu machen. Dazu wird, wie ich meine, dieses Taschenbuch von Dieter Leuschner "Grundbegriffe der Thermodynamik" beitragen.

Das Buch macht den Leser in knapper und verständlicher Form sowohl mit den Grundlagen der klassischen Thermodynamik als auch mit einigen gegenwärtigen Problemen der linearen und nichtlinearen thermodynamischen irreversiblen Prozesse bekannt. Die für die Thermodynamik charakteristischen Methoden und Verfahren der Beweisführung werden ausführlich beschrieben. Es wird kurz auf die Anwendungsgebiete der klassischen Thermodynamik und auf die Berciche, in 
denen eine Nutzung der heutigen Thermodynamik möglich ist, eingegangen. So können aus diesem Buch die Anfangskenntnisse geschöpft werden, die den $\mathrm{Zu}$ gang zur modernen Thermodynamik ermöglichen. Diese Kenntnisse sind auch für den ausreichend, der den hohen Stand der Thermodynamik für praktische Zwecke nutzen will. Ich möchte noch die besondere Bedeutung der Verbreitung der Thermodynamik in der DDR hervorheben. In der Vergangenheit schufen hervorragende deutsche Gelehrte die Grundlagen dieser Wissenschaft. Die heutige Generation der Wissenschaftler in der DDR liefert einen würdigen Beitrag zu dem sich rasch entwickelnden Zweig der Thermodynamik der nichtlinearen irreversiblen Prozesse.

Prof. A. I. Zotin

Moskau 


\section{Vorwort}

Das vorliegende Taschenbuch enthält eine Zusammenstellung und Erläuterung elementarer Grundbegriffe der Thermodynamik, deren Kenntnis weiterführende Studien ermöglicht.

Es wurde dabei der „,klassische“ (phänomenologische Theorie und anschließende statistische Begründung) und nicht der von der Informationstheorie bzw. Quantenstatistik ausgehende Weg gewählt. Die Gesetzmäßigkeiten der Thermodynamik, aufbauend auf den grundlegenden Begriffen einschließlich Maßeinheiten, sind axiomatisch in Form von Existenzsätzen formuliert. Zugehörige Erläuterungen folgen jeweils. Die statistische Thermodynamik ist anschließend kurz behandelt, während die technische Thermodynamik nur sporadisch erwähnt werden konnte. Den Abschluß bilden Anwendungsbeispiele und ein Anhang, in dem einiges gebracht ist, was sich nicht in den Rahmen von Kap. 2 einbauen ließ.

Die Methodik ist teilweise deduktiv, teilweise induktiv. Die Verwendung der Symbole erfolgte nach F. HuND: $E$ für die Energie (vgl. auch GLansdorfF und PrigoGINE; wegen der Verwendung von $E$ in der statistischen Thermodynamik, vgl. Teil 3, ist das gleiche Symbol auch in der phänomenologischen Thermodynamik benutzt) und $A$ für die Arbeit.

Zum Abschluß möchte ich denen danken, die mich bei der Entstehung des Büchleins unterstützt haben: Prof. D. S. Chernavskis, Prof. M. Eigen, Prof. I. Prigogine, Prof. A. I. Zотіл für fachliche Förderung, Prof. Dr. Dr. h. c. G. FrIEDRICH für sein langjähriges, förderndes 
Interesse, Prof. Dr. N. Elsner, Doz. Dr. P. Paufler, Dr. Schneider und Prof. Dr. H. Ulbricht für kritische Hinweise, Frau Dipl.-Phys. U. Hemmans für gute Zusammenarbeit mit dem Verlag.

Dieter Leuschner 


\section{Inhaltsverzeichnis}

\section{Einführung}

1.1. Voraussetzungen. . . . . . . . . . . . . . . . 11

1.1.1. Thermisches Vierersystem . . . . . . . . . . . . 11

1.1.2. Abgeleitete Größen . . . . . . . . . . . . . . . 13

1.1.3. Zusammensetzung . . . . . . . . . . . . . . . 14

1.2. Grundlegende thermodynamische Begriffe. . . . . 17

1.2.1. Thermodynamisches System . . . . . . . . . . 18

1.2.2. Thermodynamischer Zustand . . . . . . . . . . . 19

1.2.3. Zustandsänderungen . . . . . . . . . . . . . . 26

1.2.4. Zustandsgleichungen . . . . . . . . . . . . . . 28

1.2.5. Thermodynamische Arbeit . . . . . . . . . . . 38

\section{Thermodynamische Gesetze}

2.1. Nullter Hauptsatz (0.HS) . . . . . . . . . . . . 42

2.2. Erster Hauptsatz (1.HS) . . . . . . . . . . . . . . . 45

2.3. Zweiter Hauptsatz (2.HS) . . . . . . . . . . . . 53

2.3.1. Totales Differential $\mathrm{d} S$. . . . . . . . . . . . . 53

2.3.2. GrbBsssche Gleichung . . . . . . . . . . . . . . . 72

2.3.3. Thermodynamische Potentiale, chemisches Potential . . . . . . . . . . . . . . . . . . . . . 76

2.3.4. Gleichgewichts- und Stabilitätsbedingung . . . . . 92

2.4. Nernstsches Theorem . . . . . . . . . . . . . 102

2.5. Prinzip der minimalen Entropieproduktion . . . . 104

2.6. Nichtlinearitäten . . . . . . . . . . . . . 112

\section{Statische Thermodynamik}

3.1. Statistische Begründung thermodynamischer Gesetzmäßigkeiten . . . . . . . . . . . . . . . . 121

3.1.1. Statistik im mechanischen Phasenraum: Stationäre Gesamtheiten . . . . . . . . . . . . 121

1.1.2. Die Hauptsätze . . . . . . . . . . . . . . . . 133 
3.2. Verschiedene Statistiken . . . . . . . . . . . . 141

3.3. Statistik des Nichtgleichgewichts . . . . . . . . 147

\section{Anwendungen}

4.1. Zusammenhang von Energie und Zustandsgleichung für thermodynamische Systeme . . . . . . . . . 149

4.2. Seebeck-Effekt . . . . . . . . . . . . . . . . 152

4.3. Instabilität einer biochemischen Reaktion . . . . . 155

4.4. Absolutwert thermodynamischer Kenngrößen . . . 162

5. Anhang

5.1. Netzwerkthermodynamik . . . . . . . . . . . . 164

5.2. Relativistische Thermodynamik . . . . . . . . . 169

5.3. Hydrothermodynamik . . . . . . . . . . . . 172

5.4. Entropie und Information . . . . . . . . . . . . 173

5.5. Negative thermodynamische Temperaturen . . . . 175

Literaturverzeichnis . . . . . . . . . . . . . . 178

Sachverzeichnis . . . . . . . . . . . . . . . 181 